\title{
AN APPROACH TOWARDS BALLISTIC MISSILES PERFORMANCE IMPROVEMENT THROUGH THE APPLICATION OF HIGH-SPEED NAVIGATION PROCESSORS
S. A.Gadalla ${ }^{*}$
M.A.El-1ithy
A.H.Makariuos

ABSTRACT

NAVSTAR/GPS Global positioning system may be applied in ballistic missiles (BM) for determination of its position on the ballistic phase of its trajectory. The methods for solution of GPS system of equations are the iterative, algebraic, and direct methods. We have applied these methods for simulated ballistic trajectories. The results presented in this paper showed that the iterative one is slower but its final error is smaller. Therefore, a new modified method which is faster and less erroneous; is proposed.. The navigation algorithms are elaborated, using the modified method, and are executed by different types of microprocessors and the results are presented. The optimum processor which provides the minimum execution time is specified. Impact of the navigation processor speed on the ballistic missile-guidance process resides in the fact that a reduced execution time of the navigation algorithm provides better possibility for delivery of higher number and almost continuous generation of guidance commands to the ballistic missile along the majority of its flight trajectory within certain time interval, which will result in an improvement of its performance. The guidance process may be improved through the application of high-speed processors. A performance improvement criterion is defined and a performance improvement (PI) factor is evaluated. The results show that an improvement of about $66.7 \%$ may be attained.

1. Introduction

The present paper comprises two main parts; the first of which concerns with the application of different GPS navigation algorithms onto BM motion along its free-flight trajectory while the second introduces an approach towards BM performance improvement through the application of

* Department of Guidance, M.T.C.,Cairo,Egypt. 
different microprocessor for such GPS algorithms. Therefore, a model and a simulation of BM motion have been elaborated [4].. A new method utilizing the advantages of both the direct method and the iterative method are introduced in this paper.

\section{PART I. GPS Navigation Algorithms}

GPS navigation algorithms may be classified according to the accuracy of position mesurement and the speed of calculation.

\section{I.1. Solution of GPS Navigation Equations}

The iterative, algebraic, and direct methods are three methods used in the solution of the following GPS equations

$$
r_{i}=d\left(x, s_{i}\right)+b \quad i=1, \ldots, 4
$$

where;

$$
\begin{aligned}
& r_{i} \text { denotes the pseudorange from user to the satellite number } i \\
& x \quad \text { is the user vector position w.r.t. the inertial frame } \\
& s_{i} \quad \text { is the satellite } i \text { coordinate w.r.t the inertial frame } \\
& \mathrm{d}\left(\mathrm{x}, \mathrm{s}_{\mathrm{i}}\right) \text { is the geometric distance from the user to the satellite } i \\
& \mathrm{~b} \quad \text { is the clock offset }
\end{aligned}
$$

the iterative method $[1]$ which applies Newton method involves iterations on $4 \times 4$ augmented range direction cosine matrix beginning with an assumed position. The algebraic method [2] is nonitertive in nature. Its solution is dependent on the solution of a quadratic scalar equation resulting from equating the squares of $\left(x-s_{i}\right)$ and $\left(r_{i}-b\right)$. The direct method $[3]$ is the most recent one which uses a new system of equations expressed by satellites positions. The expressed system of equations is reduced to three linear equations and auxiliary quadratic equation dependent on the clock offset $b$. The application of this methods to ballistic missile (BM) trajectory is given in [4]. In the present paper the BM trajectory considered is started from burn-out 
point at longitude $30^{\circ}$ and geocentric latitude $30^{\circ}$ and is terminated at re-entry point of longitude $30^{\circ}$ and geocentric latitude of $35^{\circ}$. Therefore the range angle of the considered trajectory is $5^{\circ}$.

\section{I.2. Formulation of Algorithms For Solution of GPS Equations}

The receiver data which is to be processed by the GPS navigation processor is simulated on the GPS model [4]. The starting instant of the missile flight is considered zero GPS time. The formulation of algorithms for processing of such data is presented in the following sections.

\section{I.2.1. Formulation of The Iterative Method Algorithm}

Fig(1) shows the flow chart of the algorithm. It begins with an estimate of user's position. The algorithm uses the linearization of the pseudorange equations about the current estimated user position and solves successively for correction based on measuring the residuals $(\Delta r)$. In some cases the processing of the algorithm becomes divergent when the user is in the place that yields equivalent direction cosines leading to some ill condition in the determinant of the augmented direction cosine matrix. 


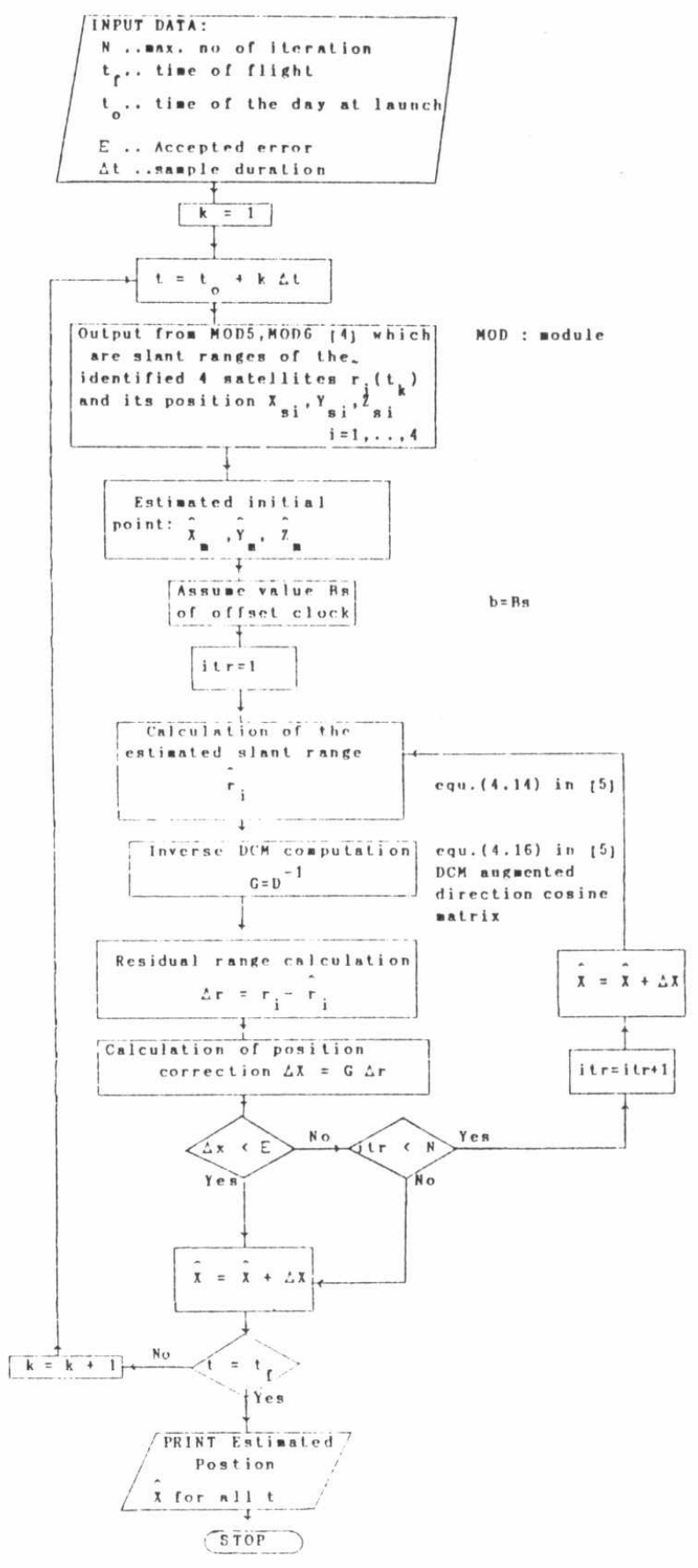

Fig(1) Iterative Method Flow chart 


\subsection{Formulation of The Algebraic Method Algorithm}

The algebraic solution depends on the solution of a quadratic scalar equation, the roots of which involve the user's position $x$, and the clock offset $b$, which is given by

$$
\lambda^{2} u^{\mathrm{T}} \beta u+2 \lambda\left(u^{\mathrm{T}} \beta v-1\right)+v^{\mathrm{T}} \beta v=0
$$

where : $\beta, \lambda, u, v$ are defined in the flow chart of fig(2).

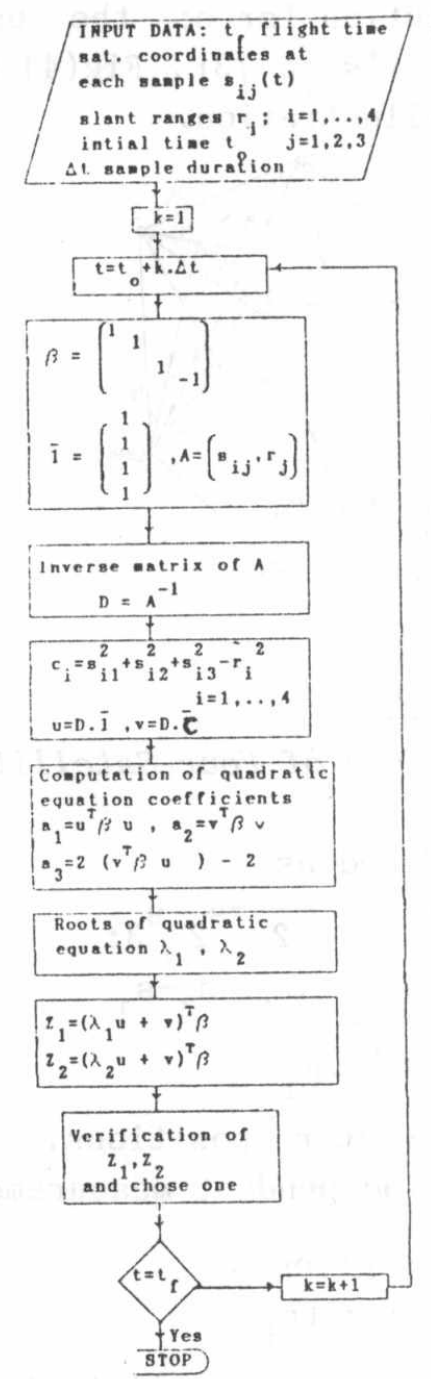

Fig(2) Algebraic Method Flow Chart 


\section{I.2.3 Formulation of The Direct Method Algorithm}

The solution using this method is based on the difference equation linearization. The set of satellite position vectors define a basis for the measurement and computation process. Two dimensions of the basis are formed from the sequential difference of the position vectors. Any adjacent two of these difference vectors form a measurement plane. The third dimension of the measurement system is formed with a unit vector normal to this plane and is derived using a direct and simple computation involving a $2 \times 2$ matrix inversion and a square root. After expressing the system of equations in the new basis, the four equations are reduced to three linear difference equations. A fourth auxiliary quadratic equation is then formulated, which provides the immediate unambiguous direct precise solution for b; the user's clock offset. This method is analyzed in details in [3]. Fig(4) shows the geometry of the four satellites used for illustration.

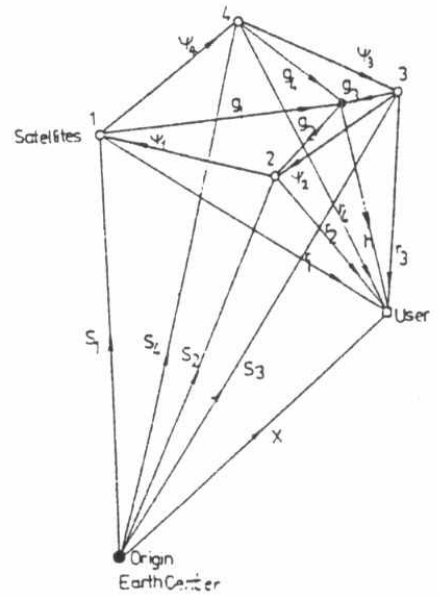

Fig(4) geometry of Four Satellites

The four basis vectors are defined as

$$
\begin{gathered}
\psi_{1}=\mathrm{s}_{1}-\mathrm{s}_{2} ; \psi_{2}=\mathrm{s}_{2}-\mathrm{s}_{3} ; \\
\psi_{3}=\mathrm{s}_{3}-\mathrm{s}_{4} ; \psi_{4}=\mathrm{s}_{4}-\mathrm{s}_{1} \\
\text { and } \sum_{\psi_{i}}=0
\end{gathered}
$$

where $\mathrm{s}($.$) are the satellites vector positions. The adjoining pairs$ $\left(\psi_{1}, w_{2}\right)$ or $\left(\psi_{3}, w_{4}\right)$, forms an independent measurement plane.

The user's position vector $x$ is given by

$$
\mathrm{x}=\mathrm{s}_{\mathrm{i}}+\mathrm{r}_{\mathrm{i}}
$$

where $r_{i}$ is the vector range from the satellite to the user. 
Again, from $f i g(4)$ the range vectors are given by

$$
r_{j}=g_{j}+h, \quad g_{j}=k_{j}^{\psi \prime}
$$

where $h$ is the orthogonal to the selected basis plane $\psi=\left[\begin{array}{l}\psi \\ j \\ \psi_{j+1}\end{array}\right]$

The unknowns to be determined are the : $_{j}$ 's, the magnitude of $|\vec{h}|$ and the user's clock of fset b.

Fig(5) shows the flow chart of the direct method algorithm. Note: all equations inside the flow chart are taken from [3]

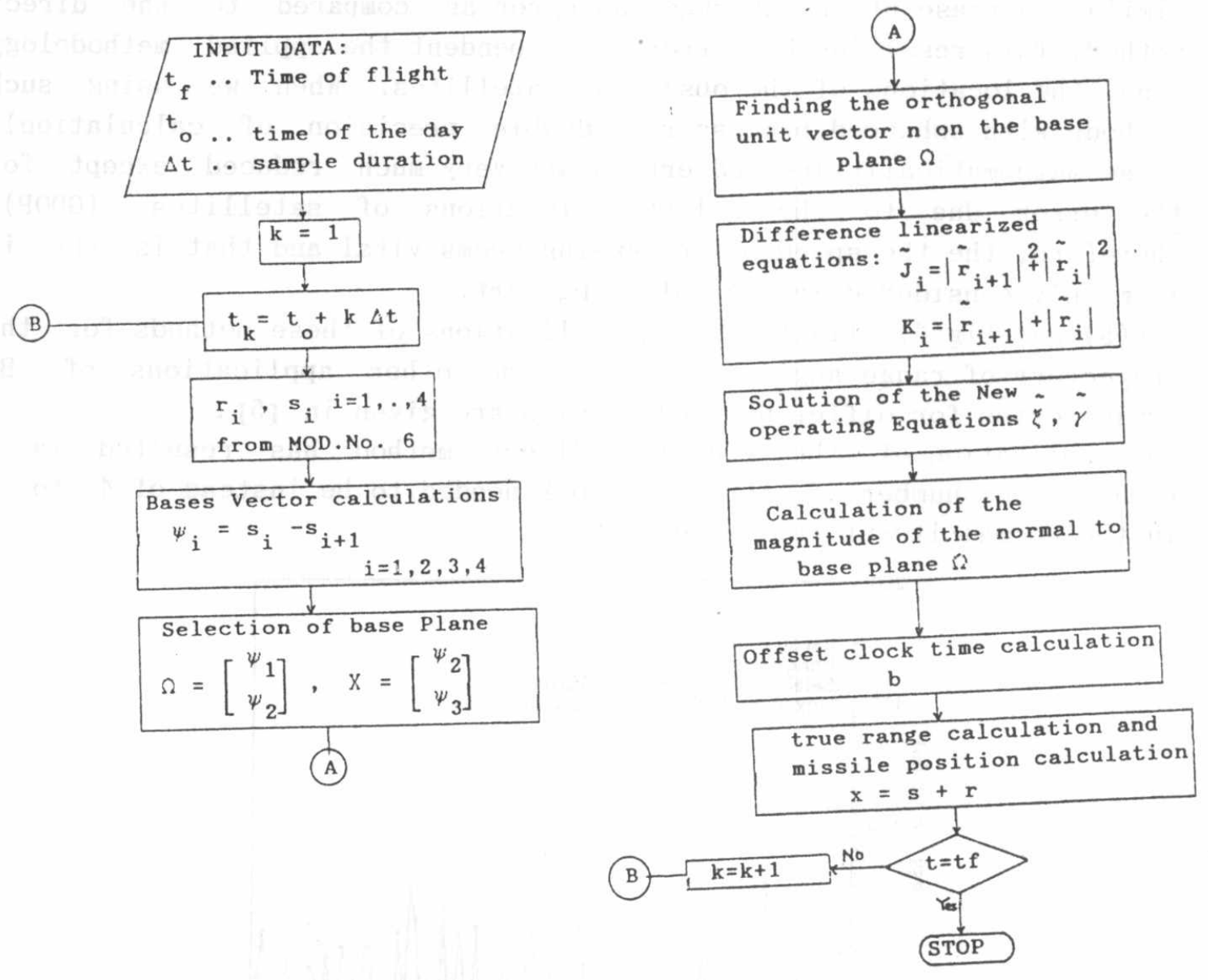

Fig(4) Direct Method Flow Chart 


\section{I.3. Evaluation of Results}

The RSS error resulting from the application of the direct method is greater than of the iterative method but the later consumes longer time. The iterative method must start with a guess of the initial state and the number of iterations required depends on the accuracy of the initial guess. We propose a modification that will reduce the RSS error in determining the position of the BM using the direct method, which we call the modified direct method. This is done by applying first the direct method and using its output as an initial guess of the state for the iterative method. We have obtained a saving in time of approximately $70 \%$ compared with the iterative method alone, and a similar decrease of the average RSS error as compared to the direct method. This resulting RSS error is dependent the applied methodology and the locations of the observed satellites. When we using such methods with enhanced processors (double precision of calculation), these mathematically induced errors are very much reduced except for the error due to the selected locations of satellites (GDOP). Therefore, the the speed of processing seems vital and that is why it is mainly considered in the following part.

Fig(5) and $f i g(6)$ illustrate the applications of these methods for the trajectory of range angle $\alpha=5$. The other applications of BM trajectories for different range angles are given in $[5]$.

The application of the modified direct method has resulted in a reduction of number of iterations to 2 needed to be instead of 4 to 6 in the normal iterative method.

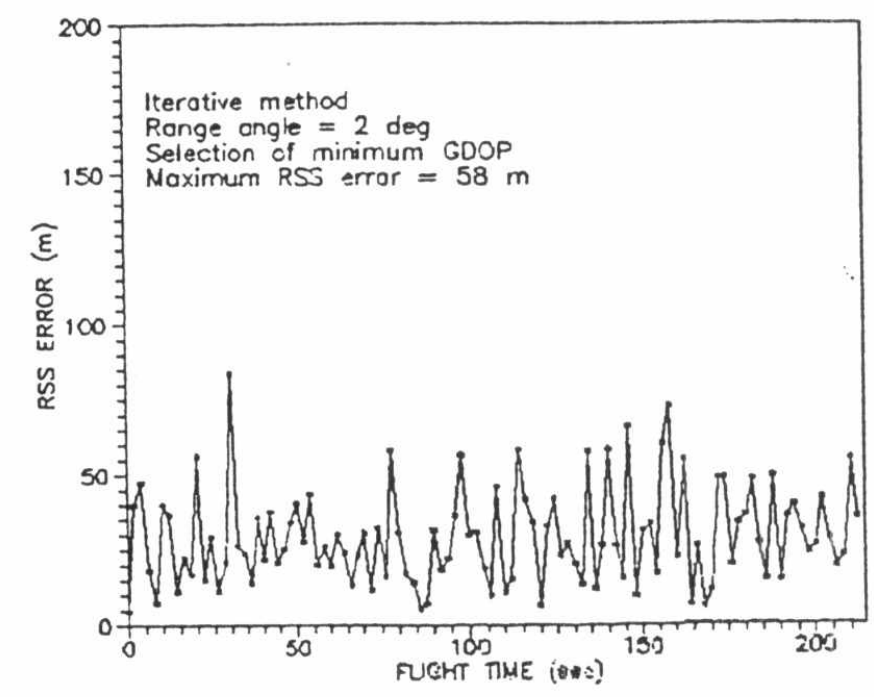

fig(5) Iterative method RSS error for $;=5^{\circ}$ 

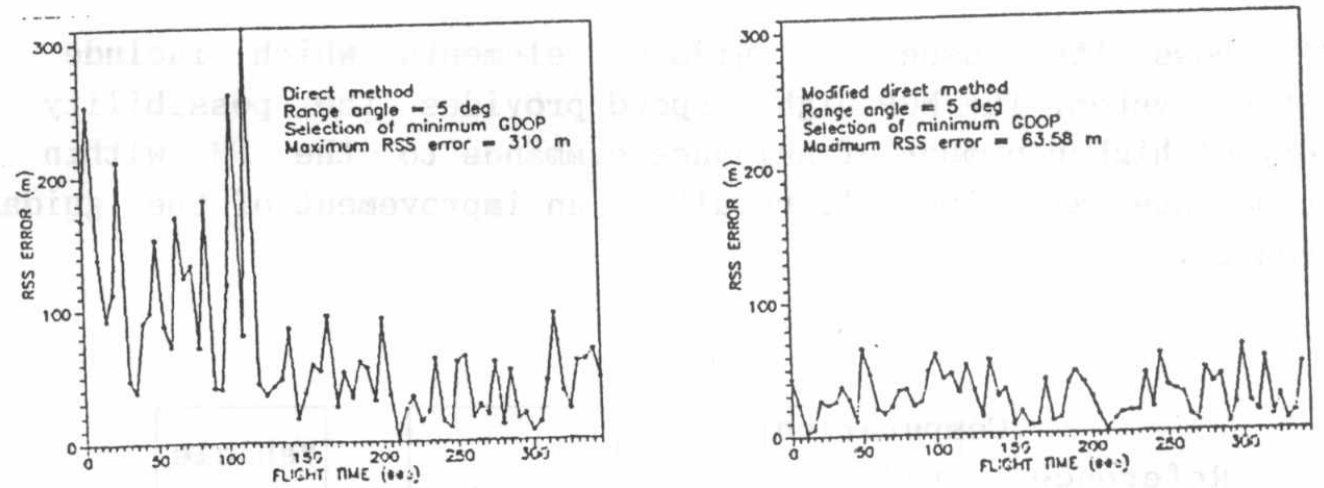

Fig(6) Direct and Modified Direct Methods RSS error, $\theta=5^{\circ}$

Fig(7) shows the average values of the previous curves by using the best exponential curve fitting.

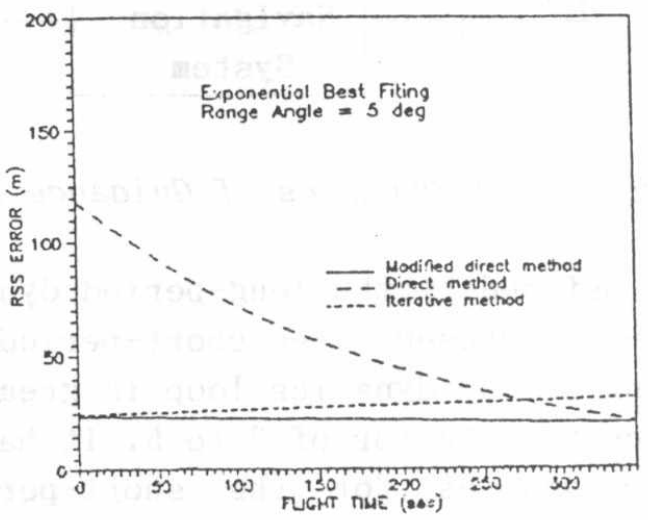

Fig(7) Exponential Fitting For GPS Methods, $;=5^{\circ}$

Part II. BM Guidance Improvement

\section{II.1. Time Limitations in Navigational Guidance Performance}

In the guidance process of $\mathrm{BM}$, there are three factors characterizing the navigation system used; namely cost, accuracy, and speed In this section we specify the accepted execution time for the GPS navigation system to be used in the BM's and the corresponding imposed limitations. The GPS navigation system comprises the receiver and a navigation processor (NGP) which are intended for determination of BM position. The guidance performance improvement resides in the determination of a higher efficient fast processing of guidance loop. 
Fig(8) shows its essential guidance elements which include the navigation system. The NGP higher speed provides the possibility for delivery of higher number of guidance commands to the BM within the same time interval which will result in an improvement of the guidance performance.

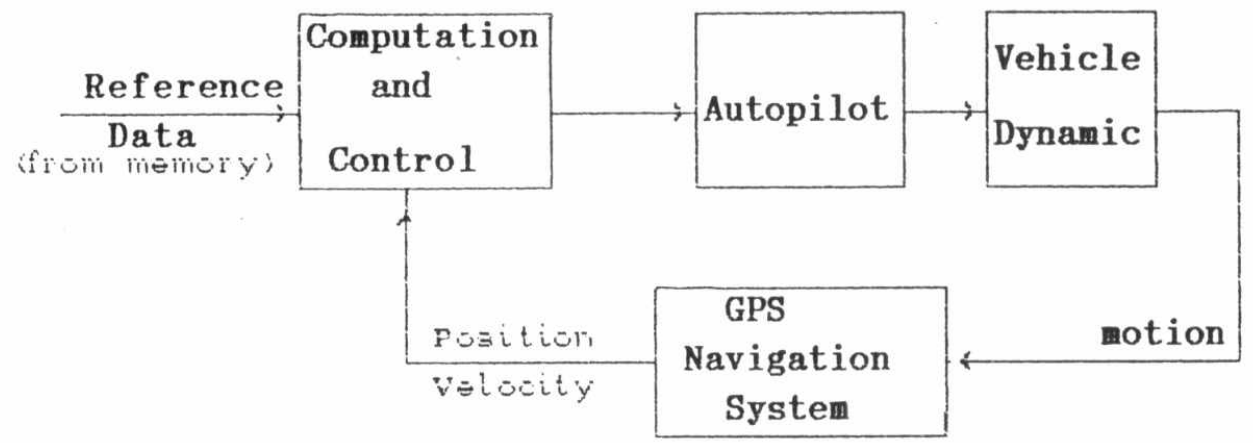

Fig(8) Long-period Dynamics of Guidance Loop

The BM guidance loop is defined by the long-period dynamics while the autopilot inside this loop represents the short-period dynamics. The time constant of the long-period dynamics loop is greater than that of the short period dynamics by a factor of 3 to 5 . It has been found that the booster vehicles frequencies for the short-period dynamics $F_{S}$ ranges from 0.067 to $100 \mathrm{rad} / \mathrm{sec}[6]$. Let $\mathrm{F}_{\mathrm{L}}$ be the frequency for the long-period dynamics and $\tau_{L}$ is the corresponding time constant; then :

$$
\tau_{\mathrm{L}} \doteq 3 \text { to } 5 \tau_{\mathrm{S}}
$$

We note that the autopilot is in charge of the stability of the control system of the missile, therefore to guarantee that the stability of the control system is completely settled, then the time constant of the long-period dynamic $\tau_{L}$ should not be less than $5 \tau_{\mathbf{s}}$. However, the worst case for the NGP execution time $t_{G}$ is when $\tau_{L}$ is taken equal to $3 \tau_{S}$ such that $\tau_{\mathrm{L}} \leq 3 \tau_{\mathrm{S}}$.

The navigation processor (NGP) is basically centered around a microprocessor; its specifications have to be well-defined to achieve the required processing time. 
The algorithms of GPS navigation methods have been elaborated and executed using different microprocessors and the results are shown in table (1). The entries of that table represent the execution time for the "receiver data processing" part of the GPS algorithms while the time of the "receiver data acquisition" part is not included. This means that the total execution time may be approximately doubled.

Table(1) Execution Times in GPS Model

\begin{tabular}{|l|l|l|l|l|}
\hline Microprocessors & $\begin{array}{l}\text { Microcomputer } \\
\text { frequency }\end{array}$ & $\begin{array}{l}\text { Direct } \\
\text { Method }\end{array}$ & $\begin{array}{l}\text { Iteative } \\
\text { Method }\end{array}$ & $\begin{array}{c}\text { Modified Direct } \\
\text { Method }\end{array}$ \\
\hline 8088 & $5 \mathrm{MHZ}$ & $6.3 \mathrm{~s}$ & $12.3 \mathrm{~s}$ & $9.6 \mathrm{~s}$ \\
\hline AT80286+Coproc. & $12 \mathrm{MHZ}$ & $0.05 \mathrm{~s}$ & $0.20 \mathrm{~s}$ & $0.1 \mathrm{~s}$ \\
\hline $80386 \mathrm{SX}$ & $12.1 \mathrm{MHZ}$ & $0.49 \mathrm{~s}$ & $1.92 \mathrm{~s}$ & $1.0 \mathrm{~s}$ \\
\hline $80386 \mathrm{SX}$ & $16 \mathrm{MHZ}$ & $0.38 \mathrm{~s}$ & $1.46 \mathrm{~s}$ & $0.8 \mathrm{~s}$ \\
\hline $80386 \mathrm{SX}$ & $24 \mathrm{MHZ}$ & $0.22 \mathrm{~s}$ & $0.94 \mathrm{~s}$ & $0.5 \mathrm{~s}$ \\
\hline 80486 & $40 \mathrm{MHZ}$ & $0.008 \mathrm{~s}$ & $0.13 \mathrm{~s}$ & $0.04 \mathrm{~s}$ \\
\hline
\end{tabular}

\section{II.2. Performance-Improvement Factor Definition and Evaluation}

From table(1), it is clear that the 80486 with clock frequency of 40 MHZ, realizes a total execution time of about $16 \mathrm{msec}$.

If we define the missile-performance improvement factor (PI) to be

$$
\text { PI }=\frac{t_{0}-t_{1}}{t_{0}} \cdot 100
$$

where $t_{0}$ : is the worst case NGP execution time which is specified by missile dynamics $\left(t_{0}=30 \mathrm{msec}\right)$.

$\mathrm{t}_{1}$ : is the actual NGP processing time (16 $\mathrm{msec}$ in our case).

In such case, the corresponding value of PI will be:

$$
\mathrm{PI}=\frac{30-16}{30} \cdot 100=46.7 \%
$$

This means that by applying such fast processor as a NGP, an improvement in the missile performance of about $46.7 \%$ is realized. Further and significant improvement in the missile performance can be attained through the application of high-speed microprogrammble microprocessors (Bit-Sliced). 


\section{II.3. Bit-Sliced Microprocessors}

Unlike single-chip microprocessors, bit sliced microprocessors divide the assigned processor functions among several IC's. The registers and ALU are packaged separately from the control. The control portion of the processor is constructed from a microprogram sequencer IC and other $\operatorname{logic}$ to decode and execute instructions $[7]$. When the microprogram sequencer decodes an instruction, it generates a starting address to the microprogram memory which is a separate package. Hence the machine language instruction set can be customized for an application. This trend is advantageous due to the following:

1) most bit sliced microprocessors are faster than single-chip MOS processors due to the application of bipolar technology.

2) the small size cascadable slices provide the designer with the capability of data wordlength adjustment to match the specific application.

3) the designer can also optimize the instruction set for the particular application.

4) application of a piplined architecture which realizes fast processing through ignoring the microinstruction fetch time.

In a previous research work $[8\}$, a bit-sliced configuration (based on the Am2900 microprocessor) has been compared to a configuration using the 80286 processor (with co-processor and $16 \mathrm{MHZ}$ ) clock). It has been found that the bit-sliced one out-performs by a factor of six times faster than the second one. Remembering that the superslice AM2903 is more powerful and faster than 2901 which was used in the mentioned comparison, we may expect that such a bit-sliced configuration (using the super-slice 2903) will be 10 times less, i.e.about $5 \mathrm{msec}$ (doubled as $10 \mathrm{msec}$ ). The improvement of the missile performance, in such case, will be :

$$
\mathrm{PI}=\frac{30-10}{30} \cdot 100=66.7 \%
$$

This means that by applying such a dedicated bit-sliced configuration an outstanding improvement in the missile performance may be attained.

Conclusion

The direct method is faster than the iterative one but the final error is greater.

The modified direct method saves $70 \%$ of the execution time of the iterative one and the resulting error is the best for the application of such GPS methods. 
When applying enhanced processors (double precision of calculation) the mathematically induced errors from these GPS navigation algorithms are very much reduced.

Indeed, as the navigation processor speed is increased, the execution time of such method is effectively reduced and the missile performance is much improved.

Bit-sliced technique may also be considered to increase the processor speed, providing a performance improvement factor of about $66.7 \%$.

\section{References}

[1] Noe,P.s and Mayer, K.A., "A Position-Fixing Algorithm For The Low Cost Receiver" IEEE Trans. on Aerospace And Elec. Sys., Vol.AES-2 No. 2,pp245-298, march (1976).

[2] Stephen Bancroft, "An Algebraic Solution of GPS Equations" IEEE Trans. on Aerospace and Elec. Sys., Vol.AES-11, No.1 jaunuary (1985).

[3] Lloyd 0.Krause,"A Direct Solution of GPS Type Navigation Equations" IEEE Trans, on Aerospace and Elec. Sys., Vol.AES-23 No.2 March (1987).

[4] S.A.Gadalla,M.A.El-lithy,A.H.Makariuos, "Simulation of Ballistic Missile Motion in Free-Flight (ballistic) phase Using NAVSTAR (GPS) Navigation Model",Fifth ASAT Conference, M.T.C. Cairo 4-6 May (1993).

[5] S.A.Gadalla,"Application of Modern Navigation Systems For Ballistic Missiles Guidance",M.Sc. Thesis, M.T.C. Cairo (1992).

[6] Arthur L.Greensite,"Analysis and Design of Space Vehicle Flight Control System" Spartar Books (1970).

[7] Kenneth L. Short, "Microprocessors and Programmed Logic", Englewood Cliffs: Prentic-Hall, Inc.(1988).

[8] A.I.Hashad, "An Enhanced System For Guidance And Control of IR Command Guided Missile", M.Sc. M.T.C. Cairo (1988). 